\title{
Intramolecular Ionic Diels-Alder Reactions of $\alpha$-Acetylenic Acetals
}

Seung-Bo Shim, Yoon-Joo Ko, Byeong-Wook Yoo, Chang-Keun Lim, Jung-Hyu Shin*

School of Chemistry, Seoul National University, Seoul 151-742, Korea

junghyu@snu.ac.kr

\section{SUPPORTING INFORMATION}

Table of contents

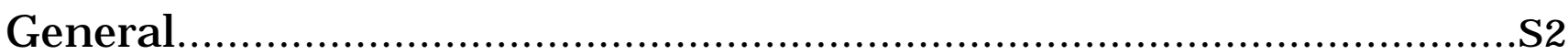

Procedures and Characterizations............................................... 2

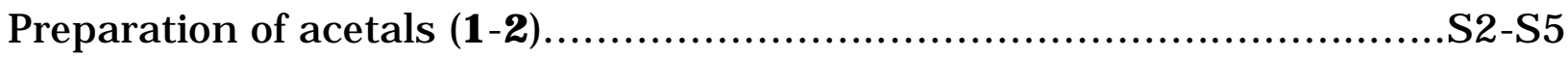

Lewis acid-catalyzed cycloaddition of $\alpha$-acetylenic acetal.........................S5

Formic acid-catalyzed cycloaddition of $\alpha$-acetylenic acetal.......................S6

Aromatization of 6-methyl-4a,7-dihydro-1-tetralone (11) .......................S7

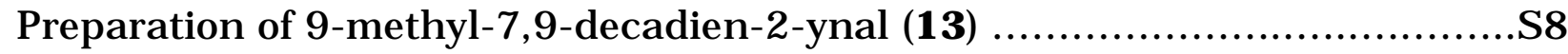

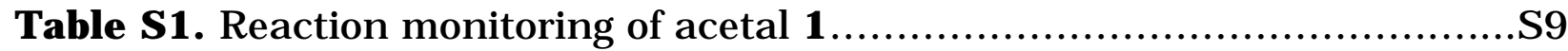

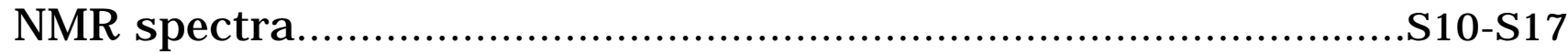

${ }^{1} \mathrm{H}$ NMR spectrum of $\mathbf{1} \ldots \ldots \ldots . . . \mathrm{S} 10 \quad{ }^{13} \mathrm{C}$ NMR spectrum of $\mathbf{1} \ldots \ldots \ldots \ldots \ldots . . . . . . .11$

${ }^{1} \mathrm{H}$ NMR spectrum of 2..........S12 $\quad{ }^{13} \mathrm{C}$ NMR spectrum of 2................S13

${ }^{1} \mathrm{H}$ NMR spectrum of $\mathbf{9} \ldots \ldots \ldots . . . \mathrm{S} 14 \quad{ }^{13} \mathrm{C}$ NMR spectrum of $\mathbf{9} \ldots \ldots \ldots \ldots \ldots . . . . . . .515$

${ }^{1} \mathrm{H}$ NMR spectrum of $\mathbf{1 1} \ldots \ldots . . . \mathrm{S} 16 \quad{ }^{13} \mathrm{C}$ NMR and DEPT spectra of $\mathbf{1 1} \ldots . . \mathrm{S} 17$ 


\section{General.}

NMR spectra were recorded at $300 \mathrm{MHz}, 500 \mathrm{MHz}$, or $600 \mathrm{MHz}$ for ${ }^{1} \mathrm{H}$, and at $75 \mathrm{MHz}$, $125 \mathrm{MHz}$, or $151 \mathrm{MHz}$ for ${ }^{13} \mathrm{C} . \mathrm{CDCl}_{3}$ was the solvent, and chemical shifts are reported in parts per million (ppm) for ${ }^{1} \mathrm{H}$ NMR on $\delta$ scale relative to TMS at $0 \mathrm{ppm}$ and for ${ }^{13 \mathrm{C}} \mathrm{NMR}$ based on the middle peak ( $\delta 77.00 \mathrm{ppm})$ of the $\mathrm{CDCl}_{3}$ solvent as an internal standard. Signal patterns are indicated as br (broad), s (singlet), d (doublet), t (triplet), q (quartet), and m (multiplet). Coupling constants $(\mathrm{J})$ are given in Hertz. Infrared (IR) spectra are reported in wave numbers (cm-1). ESI mass spectra were recorded in positive ion mode.

\section{Procedures and Characterizations.}

\section{Preparation of acetals (1-2).}

3,7-Diacetoxy-2-methyl-1-heptene (4). A $33.3 \mathrm{~mL}(16.7 \mathrm{mmol})$ solution of $0.5 \mathrm{M}$ isopropenylmagnesium bromide in THF was added dropwise to a stirred solution of aldehyde $3^{1}$ (2 g, $\left.13.9 \mathrm{mmol}\right)$ in $50 \mathrm{~mL}$ of dry $\mathrm{THF}$ at $0{ }^{\circ} \mathrm{C}$ under nitrogen. The mixture was stirred at 0 5 ${ }^{\circ} \mathrm{C}$ for $1 \mathrm{~h}$, then allowed to rise to room temperature. Acetic anhydride $(2 \mathrm{~mL}, 25.02$ mmol) was added slowly to the stirred mixture. After $5 \mathrm{~h}$ the reaction was quenched with water. The reaction mixture was evaporated under reduced pressure, then diluted with ether $(100 \mathrm{~mL})$ and washed with water $(2 \mathrm{x} 100 \mathrm{~mL})$, brine $(100 \mathrm{~mL})$, and dried over anhydrous $\mathrm{MgSO}_{4}$. The organic layer was concentrated under reduced pressure. The residue was purified by silica gel column chromatography (1:5 EtOAc/hexane) to give 4 (2.38 g, $75 \%)$ as a colorless oil.

${ }^{1}$ (a) Stowell, J. C. J. Org. Chem. 1970, 35, 244. (b) Tortajada, A.; Mestres, R.; Iglesias-Arteaga, M. A.; Synth. Commun. 2003, 33, 1809. 
TLC (EtOAc/hexane, 1:5): $\mathrm{Rf}=0.24 ;{ }^{1} \mathrm{H}$ NMR $\left(500 \mathrm{MHz}, \mathrm{CDCl}_{3}\right) \delta 5.09(\mathrm{t}, \mathrm{J}=6.73 \mathrm{~Hz}, 1 \mathrm{H})$, $4.87(\mathrm{~s}, 1 \mathrm{H}), 4.82(\mathrm{~s}, 1 \mathrm{H}), 3.98(\mathrm{t}, \mathrm{J}=6.63 \mathrm{~Hz}, 2 \mathrm{H}), 1.99(\mathrm{~s}, 3 \mathrm{H}), 1.97(\mathrm{~s}, 3 \mathrm{H}), 1.65(\mathrm{~s}, 3 \mathrm{H})$, 1.54-1.64 (m, 4H), 1.23-1.32 (m, 2H); $\left.{ }^{13} \mathrm{C} \mathrm{NMR} \mathrm{(75MHz,} \mathrm{CDCl}_{3}\right) \delta 171.1,170.2,142.9,112.8$, $76.98,64.2,32.1,28.2,21.7,21.1,20.9,18.0$.

7-Acetoxy-2-methyl-3E-1,3-heptadiene (5). According to the procedure of Tsuji et al., ${ }^{2}$ a mixture of 4 (3.01 g, $13.2 \mathrm{mmol}), \mathrm{PPh}_{3}$ (692 mg, $\left.2.64 \mathrm{mmol}\right), \mathrm{Pd}(\mathrm{OAc})_{2}$ (296 mg, $1.32 \mathrm{mmol}$ ) in freshly distilled dioxane $(20 \mathrm{~mL})$ was heated at reflux for $1 \mathrm{~h}$, at which time, analysis of a TLC indicated the absence of starting material. The suspension was filtered through a pad of Celite, and the filter cake was washed with ether. The filtrate was concentrated, dried over anhydrous $\mathrm{MgSO}_{4}$ and the residue was purified by silica gel column chromatography (1:5 EtOAc/hexane) to give 5 (2.03 g, 91\%) as a colorless oil.

TLC (EtOAc/hexane, 1:5): $\mathrm{Rf}=0.43 ;{ }^{1} \mathrm{H}$ NMR (500MHz, $\mathrm{CDCl}_{3}$ ) $\delta 6.16$ (d, J = $\left.15.64 \mathrm{~Hz}, 1 \mathrm{H}\right)$, $5.63(\mathrm{dt}, \mathrm{J}=15.64,6.99 \mathrm{~Hz}, 1 \mathrm{H}), 4.88(\mathrm{~s}, 2 \mathrm{H}), 4.79(\mathrm{t}, \mathrm{J}=6.64 \mathrm{~Hz}, 2 \mathrm{H}), 2.19(\mathrm{q}, \mathrm{J}=7.2,2 \mathrm{H})$, 2.05 (s, 3H), 1.82 (s, 3H), 1.75 (f, J = 6.8 Hz, 2H); $\left.{ }^{13} \mathrm{C} \mathrm{NMR} \mathrm{(75MHz,} \mathrm{CDCl}_{3}\right) \delta 171.2,141.9$, $133.7,129.1,114.8,64.0,29.1,28.3,21.0,18.6$.

7-Iodo-2-methyl-3E-1,3-heptadiene (6). ${ }^{3}$ After the deprotection of ester 5 under basic conditions $\left(\mathrm{K}_{2} \mathrm{CO}_{3} / \mathrm{H}_{2} \mathrm{O} / \mathrm{MeOH}\right)$, the resulting alcohol 4 was transformed into iodide 6 according to Garegg's procedure. ${ }^{5}$ A solution of 7-hydroxy-2-methyl-3E-1,3-heptadiene (147 $\mathrm{mg}, 1.16 \mathrm{mmol}$ ) in solvent (THF:acetonitrile $=1: 3,5 \mathrm{~mL}$ ) was added slowly to a stirred solution of iodine $(1.49 \mathrm{~g}, \quad 5.82 \mathrm{mmol})$, imidazol $(460 \mathrm{mg}, 6.73 \mathrm{mmol})$ and

\footnotetext{
2 (a) Tsuji, J.; Yamakawa, T.; Kaito, M.; Mandai, T. Tetrahedron Lett. 1978, 19, 2075. (b) Hauser, F. M.; Tommasi, R.; Hewawasam, P.; Rho, Y. S. J. Org. Chem. 1988, 53, 4886.

3 Cramer, C. J.; Harmata, M.; Rashatasakhon, P. J. Org. Chem. 2001, 66, 5641.

4 Nonoshita, K.; Banno, H.; Maruoka, K.; Yamamoto, H. J. Am. Chem. Soc. 1990, 112, 316.

${ }^{5}$ Garegg, P. J.; Samuelsson, B. J. Chem. Soc., Perkin trans. 1, 1980, 2866.
} 
triphenylphosphine $(1.69 \mathrm{~g}, 6.38 \mathrm{mmol})$ in solvent $(\mathrm{THF}$ :acetonitrile $=1: 3,15 \mathrm{~mL})$ at room temperature. The mixture was stirred at room temperature for $1 \mathrm{~h}$. After completion of the reaction, an aqueous sodium thiosulfate $0.1 \mathrm{M}$ solution $(50 \mathrm{~mL})$ was added dropwise to the resulting solution. The mixture was diluted with ether $(100 \mathrm{~mL})$ and washed with water $(2 \mathrm{x}$ $100 \mathrm{~mL})$, brine $(100 \mathrm{~mL})$. The ethereal layer was dried over anhydrous $\mathrm{MgSO}_{4}$ and then concentrated under reduced pressure. The residue was purified by silica gel column chromatography (hexane) to give 6 (240 mg, 82\% over two steps) as a colorless oil.

TLC (hexane): $\mathrm{Rf}=0.41 ;{ }^{1} \mathrm{H}$ NMR $\left(500 \mathrm{MHz}, \mathrm{CDCl}_{3}\right) \delta 6.20(\mathrm{~d}, \mathrm{~J}=15.62 \mathrm{~Hz}, 1 \mathrm{H}), 5.58$ (dt, J = 15.62, $7.03 \mathrm{~Hz}, 1 \mathrm{H}), 4.90(\mathrm{~s}, 2 \mathrm{H}), 3.19(\mathrm{t}, \mathrm{J}=6.94 \mathrm{~Hz}, 2 \mathrm{H}), 2.23(\mathrm{~m}, 2 \mathrm{H}), 1.94(\mathrm{~m}, 2 \mathrm{H}), 1.83$ (s, 3H); ${ }^{13} \mathrm{C}$ NMR $\left(75 \mathrm{MHz}, \mathrm{CDCl}_{3}\right) \delta 141.8,134.3,128.1,115.0,33.3,33.0,18.6,6.4$.

10,10-Diethoxy-2-methyl-deca-3E-1,3-dien-8-yne (1). According to the procedure of Chong et al., 6 to a stirred solution of propargyl aldehyde diethylacetal (7) (0.15 mL, 1.05 mmol) in dry THF (5 mL) was added 1.4 M solution of MeLi in ether (1.59 mL, $2.22 \mathrm{mmol})$ at $0{ }^{\circ} \mathrm{C}$. The resulting pale yellow solution was stirred at $0{ }^{\circ} \mathrm{C}$ for 10 minutes. $6(210 \mathrm{mg}, 0.89$ mmol) was then added followed by dry DMSO $(5 \mathrm{~mL})$. The mixture was allowed to warm to room temperature and was stirred at ambient temperature for $3 \mathrm{~h}$. The mixture was cooled in a cold water bath and quenched with the saturated brine. Ether (100 mL) was added. The organic layer was washed with $3 \times 50 \mathrm{~mL}$ of half-saturated brine and then dried over anhydrous $\mathrm{MgSO}_{4}$. The organic layer was concentrated under reduced pressure. The residue was purified by silica gel column chromatography (1:20 EtOAc/hexane) to give 1 (190 mg, 90\%) as a colorless oil.

${ }^{6}$ Chong, J. M.; Wong, S. Tetrahedron Lett. 1986, 27, 5445. 
TLC (EtOAc/hexane, 1:10): $\mathrm{Rf}=0.54 ;{ }^{1} \mathrm{H}$ NMR (500MHz, $\mathrm{CDCl}_{3}$ ) $\delta 6.08$ (d, J = $\left.15.64 \mathrm{~Hz}, 1 \mathrm{H}\right)$, 5.54 (dt, J = 15.64, $6.97 \mathrm{~Hz}, 1 \mathrm{H}), 5.19$ (s, 1H), 4.80 (s, 2H), 3.67 (f, J = $2.21 \mathrm{~Hz}, 2 \mathrm{H}$ ), 3.50 (f, $\mathrm{J}=2.21 \mathrm{~Hz}, 2 \mathrm{H}), 2.19(\mathrm{t}, \mathrm{J}=6.08 \mathrm{~Hz}, 2 \mathrm{H}), 2.14(\mathrm{q}, \mathrm{J}=7.21 \mathrm{~Hz}, 2 \mathrm{H}), 1.75(\mathrm{~s}, 3 \mathrm{H}), 1.58$ (f, J $=7.3 \mathrm{~Hz}, 2 \mathrm{H}), 1.17(\mathrm{t}, \mathrm{J}=7.13 \mathrm{~Hz}, 6 \mathrm{H}) ;{ }^{13} \mathrm{C} \mathrm{NMR}\left(75 \mathrm{MHz}, \mathrm{CDCl}_{3}\right) \delta 141.9,133.7,129.4$, 114.6, 91.4, 86.0, 76.0, 60.6, 31.7, 28.0, 18.6, 18.1, 15.1; ESI-MS: $m / z 259$ [M + $\left.\mathrm{Na}^{+}\right]$

2-(8-Methyl-nona-6E-6,8-dien-1-ynyl)-1,3-dioxolane (2). This compound was prepared from 2-ethynyl-1,3-dioxolane $(\mathbf{8})^{7}$ in a manner similar to that described for the compound $\mathbf{1}$ (46\% yield).

TLC (EtOAc/hexane, 1:10): Rf = 0.38; ${ }^{1} \mathrm{H}$ NMR (600MHz, $\left.\mathrm{CDCl}_{3}\right) \delta 6.16(\mathrm{~d}, \mathrm{~J}=15.66 \mathrm{~Hz}, 1 \mathrm{H})$, $5.62(\mathrm{~s}, 1 \mathrm{H}), 5.61(\mathrm{~m}, 1 \mathrm{H}), 4.87(\mathrm{~s}, 2 \mathrm{H}), 4.06(\mathrm{~m}, 2 \mathrm{H}), 3.92(\mathrm{~m}, 2 \mathrm{H}), 2.25(\mathrm{t}, \mathrm{J}=6 \mathrm{~Hz}, 2 \mathrm{H})$, $2.20(\mathrm{q}, \mathrm{J}=6 \mathrm{~Hz}, 2 \mathrm{H}), 1.83(\mathrm{~s}, 3 \mathrm{H}), 1.65$ (q, J = $6 \mathrm{~Hz}, 2 \mathrm{H}) ;{ }^{13} \mathrm{C} \mathrm{NMR}\left(125 \mathrm{MHz}, \mathrm{CDCl}_{3}\right) \delta 142.0$, 133.7, 129.3, 114.7, 93.2, 86.4, 76.2, 64.4, 31.7, 27.9, 18.6, 18.1; ESI-MS: m/z 207 [M + $\left.\mathrm{H}^{+}\right]$.

\section{Lewis acid-catalyzed cycloaddition of $\alpha$-acetylenic acetal.}

Procedure : Treatment of $\mathrm{TiCl}_{2}(\mathrm{O}-\mathrm{iPr})_{2}$ in $\mathrm{CH}_{2} \mathrm{Cl}_{2}$

To a solution of $\mathbf{1}(60 \mathrm{mg}, 0.25 \mathrm{mmol})$ in dry $\mathrm{CH}_{2} \mathrm{Cl}_{2}(5 \mathrm{~mL})$ at $-78{ }^{\circ} \mathrm{C}$ under $\mathrm{N}_{2}$ atmosphere was added a $3 \mathrm{M}$ toluene solution of $\mathrm{TiCl}_{2}(\mathrm{O}-\mathrm{iPr})_{2}(84 \mu \mathrm{L}, 0.25 \mathrm{mmol})$. The mixture was stirred for $0.5 \mathrm{~h}$ at $-78{ }^{\circ} \mathrm{C}$. On the completion of the reaction, the mixture was quenched with triethylamine and then concentrated under reduced pressure. The residue was diluted with ether $(30 \mathrm{~mL})$ and washed with water $(2 \times 30 \mathrm{~mL})$, brine $(30 \mathrm{~mL})$. The ethereal layer was

7 (a) Giusti, G. Bull. Chim. Soc. Fr. 1972, 753. (b) Le Coq, A.; Gorgues, A. Organic Syntheses; Wiley: New York, 1988; Collect. Vol. 6, p 954. 
dried over anhydrous $\mathrm{MgSO}_{4}$ and then concentrated under reduced pressure. The residue was purified by silica gel column chromatography (1:20 EtOAc/hexane) to give 10 (10 mg, $25 \%)$ as a colorless oil.

\section{6-Methyl-4-indancarbaldehyde (10).}

TLC (EtOAc/hexane, 1:10): Rf = 0.52; ${ }^{1} \mathrm{H}$ NMR (300MHz, $\left.\mathrm{CDCl}_{3}\right) \delta 10.05$ (s, 1H), 7.36 (s, 1H),

$7.22(\mathrm{~s}, 1 \mathrm{H}), 3.16(\mathrm{t}, \mathrm{J}=7.5 \mathrm{~Hz}, 2 \mathrm{H}), 2.82(\mathrm{t}, \mathrm{J}=7.5 \mathrm{~Hz}, 2 \mathrm{H}), 2.32(\mathrm{~s}, 3 \mathrm{H}), 2.06(\mathrm{q}, \mathrm{J}=7.5 \mathrm{~Hz}$, 2H); ${ }^{13} \mathrm{C} \mathrm{NMR}\left(75 \mathrm{MHz}, \mathrm{CDCl}_{3}\right) \delta 193.3,146.8,144.0,136.9,132.4,131.3,129.9,32.3,31.7$, 25.8, 21.4; ESI-MS: $m / z 161\left[\mathrm{M}+\mathrm{H}^{+}\right]$.

\section{Formic acid-catalyzed cycloaddition of $\alpha$-acetylenic acetal.}

Procedure A: Treatment of $0.1 \%$ formic acid in chloroform at $50{ }^{\circ} \mathrm{C}$.

To a solution of $\mathbf{1}(90 \mathrm{mg}, 0.38 \mathrm{mmol})$ in $\mathrm{CHCl}_{3}(18 \mathrm{~mL})$ was added $\mathrm{HCOOH}(18 \mu \mathrm{L}$, $0.47 \mathrm{mmol})$. The mixture was heated up to $50{ }^{\circ} \mathrm{C}$ with a charging reflux condenser. The reaction was monitored by TLC and completed in about 30 hours. The mixture was further stirred for $18 \mathrm{~h}$ at $50{ }^{\circ} \mathrm{C}$. The mixture was quenched with aqueous $\mathrm{NaHCO}_{3}$, and diluted with ether (100 mL). The ethereal solution was washed with water $(2 \times 100 \mathrm{~mL})$, brine $(100$ $\mathrm{mL}$ ), dried over anhydrous $\mathrm{MgSO}_{4}$ and concentrated under reduced pressure. The residue was purified by column chromatography on silica gel (1:20 EtOAc/hexane) to give 10 (58 mg, 95\%) as a colorless oil.

Procedure B: Treatment of $10 \%$ formic acid in pentane at $\mathrm{rt}$.

To a solution of 1 (88 $\mathrm{mg}, 0.37 \mathrm{mmol})$ in pentane $14.4 \mathrm{~mL}$ was added $\mathrm{HCOOH} \mathrm{(1.6} \mathrm{mL),}$ and the solution was stirred at room temperature. After 30 minutes, the mixture was quenched with aqueous $\mathrm{NaHCO}_{3}$ and diluted with ether $(100 \mathrm{~mL})$. The ethereal solution was 
washed with water $(2 \times 100 \mathrm{~mL})$, brine $(100 \mathrm{~mL})$, dried over anhydrous $\mathrm{MgSO}_{4}$ and concentrated under reduced pressure. The residue was purified by column chromatography on silica gel (1:20 EtOAc/hexane) to give 11 (52 mg, 86\%) as a colorless oil.

\section{6-Methyl-5,7a-dihydro-4-indancarbaldehyde (9).}

TLC (EtOAc/hexane, 1:10): Rf = 0.44; ${ }^{1} \mathrm{H}$ NMR (600MHz, $\left.\mathrm{CDCl}_{3}\right) \delta 9.94(\mathrm{~s}, 1 \mathrm{H}), 5.50(\mathrm{~s}, 1 \mathrm{H})$, $3.02(\mathrm{~m}, 1 \mathrm{H}), 2.91(\mathrm{dd}, \mathrm{J}=20.79 \mathrm{~Hz}, \mathrm{~J}=5.66 \mathrm{~Hz}, 1 \mathrm{H}), 2.86(\mathrm{~m}, 2 \mathrm{H}), 2.58$ (dd, J = 20.63 Hz, $\mathrm{J}=10.85 \mathrm{~Hz}, 1 \mathrm{H}), 2.14(\mathrm{~m}, 1 \mathrm{H}), 1.95(\mathrm{~m}, 1 \mathrm{H}), 1.79(\mathrm{~m}, 1 \mathrm{H}), 1.77(\mathrm{~s}, 3 \mathrm{H}), 1.26(\mathrm{~m}, 1 \mathrm{H}) ;{ }^{13} \mathrm{C}$ $\operatorname{NMR}\left(75 \mathrm{MHz}, \mathrm{CDCl}_{3}\right) \delta 190.7,166.0,133.3,128.3,120.5,45.3,31.7,28.7,26.9,23.7$, 22.7; ESI-MS: $m / z 163\left[\mathrm{M}+\mathrm{H}^{+}\right]$.

\section{6-Methyl-4a,7-dihydro-1-tetralone (11).}

TLC (EtOAc/hexane, 1:10): Rf =0.37; ${ }^{1} \mathrm{H}$ NMR $\left(600 \mathrm{MHz}, \mathrm{CDCl}_{3}\right) \delta 6.60$ (dd, $\mathrm{J}=6.3 \mathrm{~Hz}, \mathrm{~J}=$ $3.66 \mathrm{~Hz}, 1 \mathrm{H}), 5.35(\mathrm{~m}, 1 \mathrm{H}), 2.97(\mathrm{~m}, 1 \mathrm{H}), 2.74$ (dd, J = 9.24 Hz, J = 3.66 Hz, 2H), 2.57 (dd, J $=17.10 \mathrm{~Hz}, \mathrm{~J}=2.61 \mathrm{~Hz}, 1 \mathrm{H}$ ), 2.31 (ddd, $\mathrm{J}=17.10 \mathrm{~Hz}, \mathrm{~J}=12.60 \mathrm{~Hz}, \mathrm{~J}=6.67 \mathrm{~Hz}, 1 \mathrm{H}$ ), 2.01 $(\mathrm{m}, 2 \mathrm{H}), 1.85(\mathrm{~m}, 1 \mathrm{H}), 1.70(\mathrm{~s}, 3 \mathrm{H}), 1.46(\mathrm{dd}, \mathrm{J}=12.66 \mathrm{~Hz}, \mathrm{~J}=3.45 \mathrm{~Hz}, 1 \mathrm{H}) ; 13 \mathrm{C}$ NMR $\left(75 \mathrm{MHz}, \mathrm{CDCl}_{3}\right) \delta 202.1,138.8,131.2,130.2,123.6,40.5,38.5,32.3,32.1,23.5,23.0 ; \mathrm{IR}$ (KBr): 3012, 2925, 1693, 1674, $1622 \mathrm{~cm}^{-1}$; ESI-MS: m/z $163\left[\mathrm{M}+\mathrm{H}^{+}\right]$.

\section{Aromatization of 6-methyl-4a,7-dihydro-1-tetralone (11).}

To a solution of 6-Methyl-4a,7-dihydro-1-tetralone (11) (40 mg, $0.24 \mathrm{mmol})$ in $\mathrm{CH}_{2} \mathrm{Cl}_{2}(12$ $\mathrm{mL})$ was added DDQ $(67 \mathrm{mg}, 0.29 \mathrm{mmol})$, and then the solution was stirred at rt. After 10 minutes, the solvent was removed and the residue was filtered through a layer of $\mathrm{Al}_{2} \mathrm{O}_{3}$ (activity I) with 20\% EtOAc in hexane as eluent. The filtrate was concentrated under reduced pressure. The resulting residue was purified by column chromatography on silica gel (1:10 EtOAc/hexane) to give 12 (35 mg, 89\%) as a colorless oil. 


\section{6-Methyl-1-tetralone (12).}

TLC (EtOAc/hexane, 1:10): Rf = 0.27; ${ }^{1} \mathrm{H}$ NMR (600MHz, $\left.\mathrm{CDCl}_{3}\right) \delta 7.93(\mathrm{~d}, \mathrm{~J}=7.98 \mathrm{~Hz}, 1 \mathrm{H})$, $7.11(\mathrm{~d}, \mathrm{~J}=7.98 \mathrm{~Hz}, 1 \mathrm{H}), 7.06(\mathrm{~s}, 1 \mathrm{H}), 2.92(\mathrm{t}, \mathrm{J}=6.06 \mathrm{~Hz}, 2 \mathrm{H}), 2.63(\mathrm{t}, \mathrm{J}=6.42 \mathrm{~Hz}, 2 \mathrm{H})$, $2.38(\mathrm{~s}, 3 \mathrm{H}), 2.11(\mathrm{tt}, \mathrm{J}=6.42 \mathrm{~Hz}, \mathrm{~J}=6.06 \mathrm{~Hz}, 2 \mathrm{H}) ;{ }^{13} \mathrm{C} \mathrm{NMR}\left(125 \mathrm{MHz}, \mathrm{CDCl}_{3}\right) \delta 198.2$, 144.6, 144.2, 130.3, 129.2, 127.6, 127.3, 39.1, 29.7, 23.3, 21.7; ESI-MS: m/z $161\left[\mathrm{M}+\mathrm{H}^{+}\right]$.

\section{Preparation of 9-methyl-7,9-decadien-2-ynal (13).}

A solution of 1 (44mg, $0.19 \mathrm{mmol}$ ) in 10\% (v/v) HCOOH in acetone (200 mL) was stirred for $8 \mathrm{~h}$ at $\mathrm{rt}$. The reaction mixture was poured into EtOAc and washed with saturated aqueous $\mathrm{NaHCO}_{3}$, water, and brine. The organic layer was dried over anhydrous $\mathrm{MgSO}_{4}$ and concentrated under reduced pressure. The residue was purified by column chromatography on silica gel (1:20 EtOAc/hexane) to give 13 (13 mg, 43\%) and 9 (10 mg, 33\%), both as colorless oils, in that order of elution.

${ }^{1} \mathrm{H}$ NMR (600MHz, $\left.\mathrm{CDCl}_{3}\right) \delta 9.19(\mathrm{~s}, 1 \mathrm{H}), 6.18(\mathrm{~d}, \mathrm{~J}=15.37,1 \mathrm{H}), 5.60$ (dt, $\mathrm{J}=15.37,7.41$, $1 \mathrm{H}), 4.90(\mathrm{~s}, 2 \mathrm{H}), 2.43(\mathrm{t}, \mathrm{J}=7.14,2 \mathrm{H}), 2.24(\mathrm{~m}, 2 \mathrm{H}), 1.83(\mathrm{~s}, 3 \mathrm{H}), 1.73(\mathrm{~m}, 2 \mathrm{H})$;

ESI-MS: $m / z 163\left[\mathrm{M}+\mathrm{H}^{+}\right]$. 
Table S1. Reaction monitoring of acetal 1 in 1:10 (v/v) $\mathrm{HCOOH} / \mathrm{CDCl}_{3}$ at $25^{\circ} \mathrm{C}$

\begin{tabular}{|c|c|c|c|c|c|c|}
\hline \multirow{2}{*}{ Entry } & \multirow{2}{*}{ Time } & \multicolumn{5}{|c|}{ Yield $^{a},(\%)$} \\
\hline & & 1 & 13 & 9 & 10 & 11 \\
\hline 1 & $10 \mathrm{~min}$ & 71 & 21 & 2 & 0 & 0 \\
\hline 2 & $40 \min$ & 26 & 40 & 14 & 0 & 2 \\
\hline 3 & $1 \mathrm{~h} 20 \mathrm{~min}$ & 7 & 34 & 31 & $<1$ & 6 \\
\hline 4 & $2 \mathrm{~h}$ & 2 & 23 & 43 & 1 & 10 \\
\hline 5 & $5 \mathrm{~h}$ & 0 & 1 & 51 & 5 & 13 \\
\hline 6 & $111 \mathrm{~h}$ & 0 & 0 & $<1$ & 53 & 13 \\
\hline \multicolumn{7}{|c|}{$\begin{array}{l}{ }^{a} \text { Determined by }{ }^{1} \mathrm{H} \text { NMR using tetraethylene } \\
\text { glycol dimethyl ether as an internal standard. The } \\
\text { amounts of cyclized products were quantified by } \\
\text { comparison of the integrations of the signals at } 5.34 \\
\text { ppm for } \mathbf{1}, 9.16 \mathrm{ppm} \text { for } \mathbf{1 3}, 9.84 \mathrm{ppm} \text { for } \mathbf{9}, 10.06 \\
\text { ppm for } \mathbf{1 0} \text { and } 6.70 \mathrm{ppm} \text { for } \mathbf{1 1} \text { with that of the } \\
\text { signal at } 3.43 \mathrm{ppm} \text { for the internal standard. }\end{array}$} \\
\hline
\end{tabular}



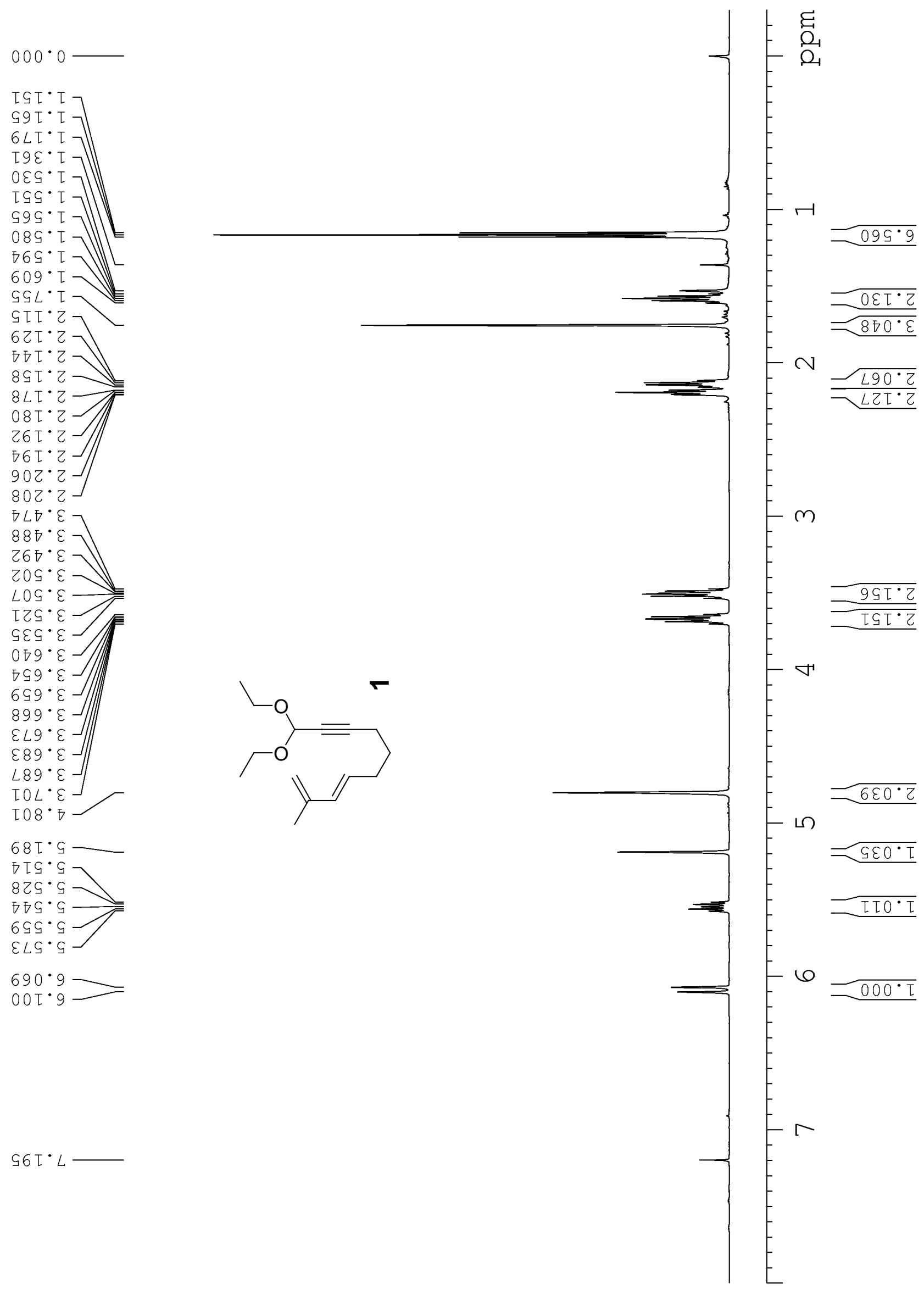

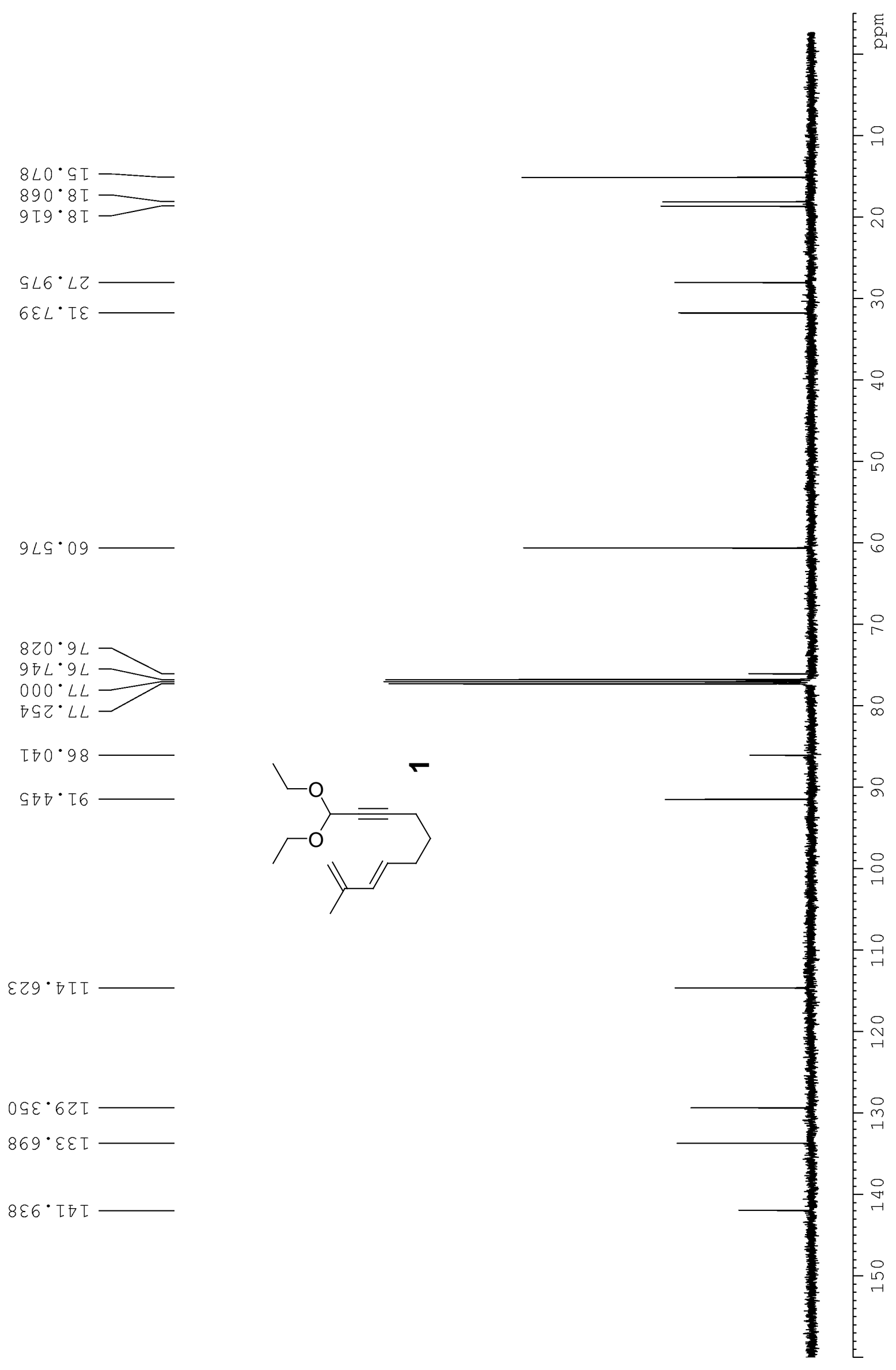

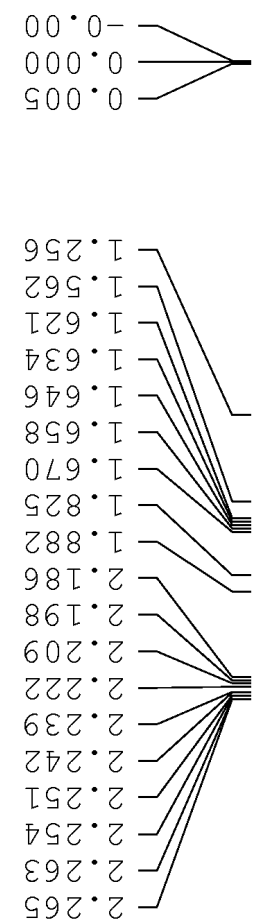

$068^{\circ}$

$906^{\circ} \varepsilon-$

Dโ6

$L \mathrm{~L} 6^{\circ} \varepsilon$

$6 \tau 6^{\circ} \varepsilon$

$\varepsilon 26^{\circ} \varepsilon$

$626^{\circ} \varepsilon$

$9 \nabla 6^{\circ} \varepsilon$

$\varepsilon \varepsilon 0^{\circ}$

โSO०

$990^{\circ} \overline{ }$

โ90

$\varepsilon 90^{\circ} \bar{\sigma}$

$990^{\circ} \nabla-$

$\square \angle 0^{\circ} \circ-$
$680^{\circ}$

$S \angle 8^{\circ} \bar{\sigma}$

$989^{\circ} 9$

$469^{\circ} 9$

โT9. 9

$929^{\circ} 9$

ง $\varepsilon 9^{\circ} \varsigma$

SDT 9

LLI.9

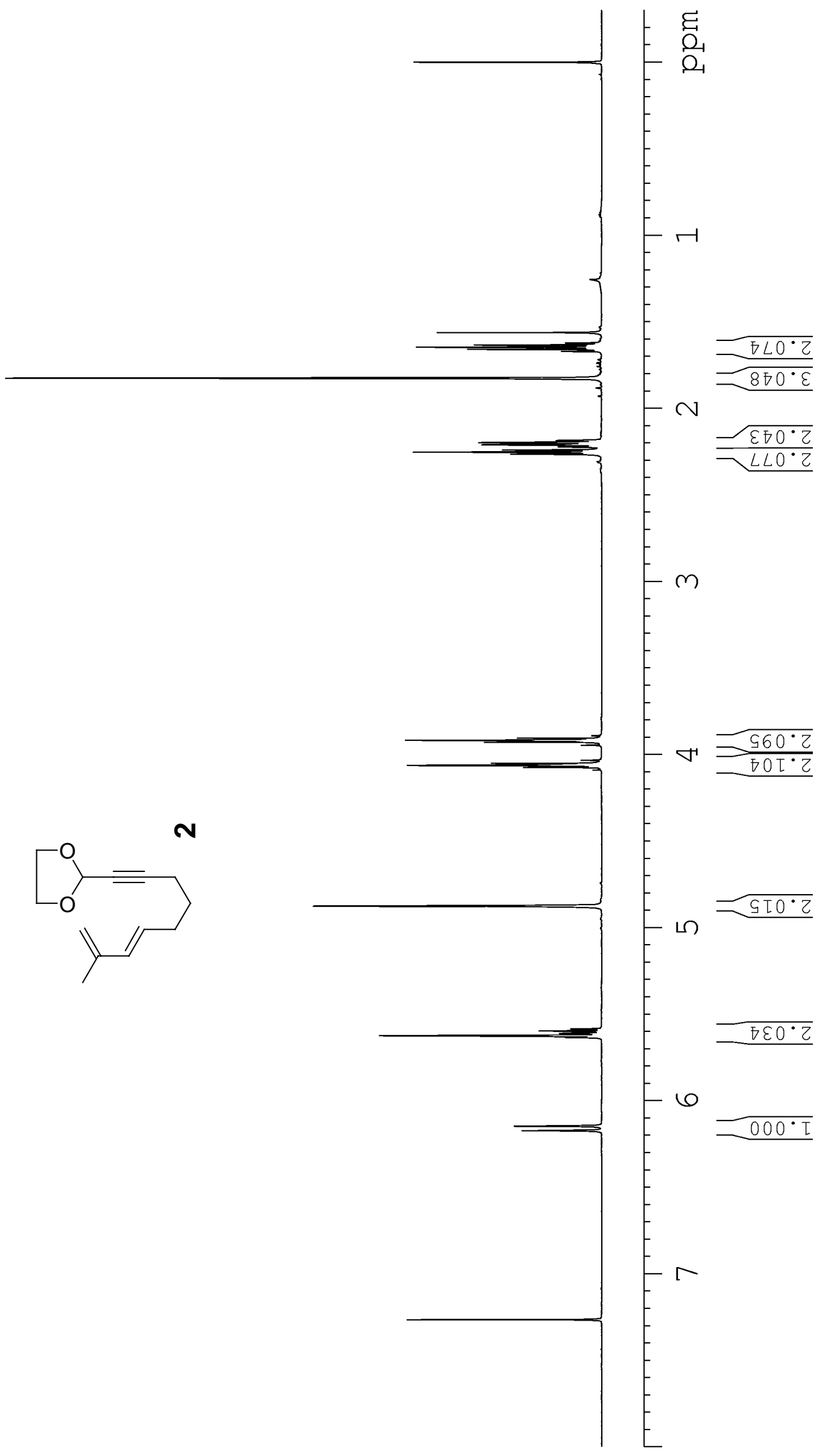

$\varepsilon 9 \tau^{\circ} L$ 
$\angle 70.81=$
829.81

$298^{\circ} \angle 2$

I89 IE

$8 E \nabla \cdot \nabla 9$

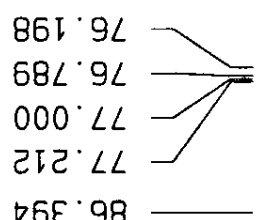

$\triangleright 6 \varepsilon^{\circ} 98$

99 'E6

gI 'E6
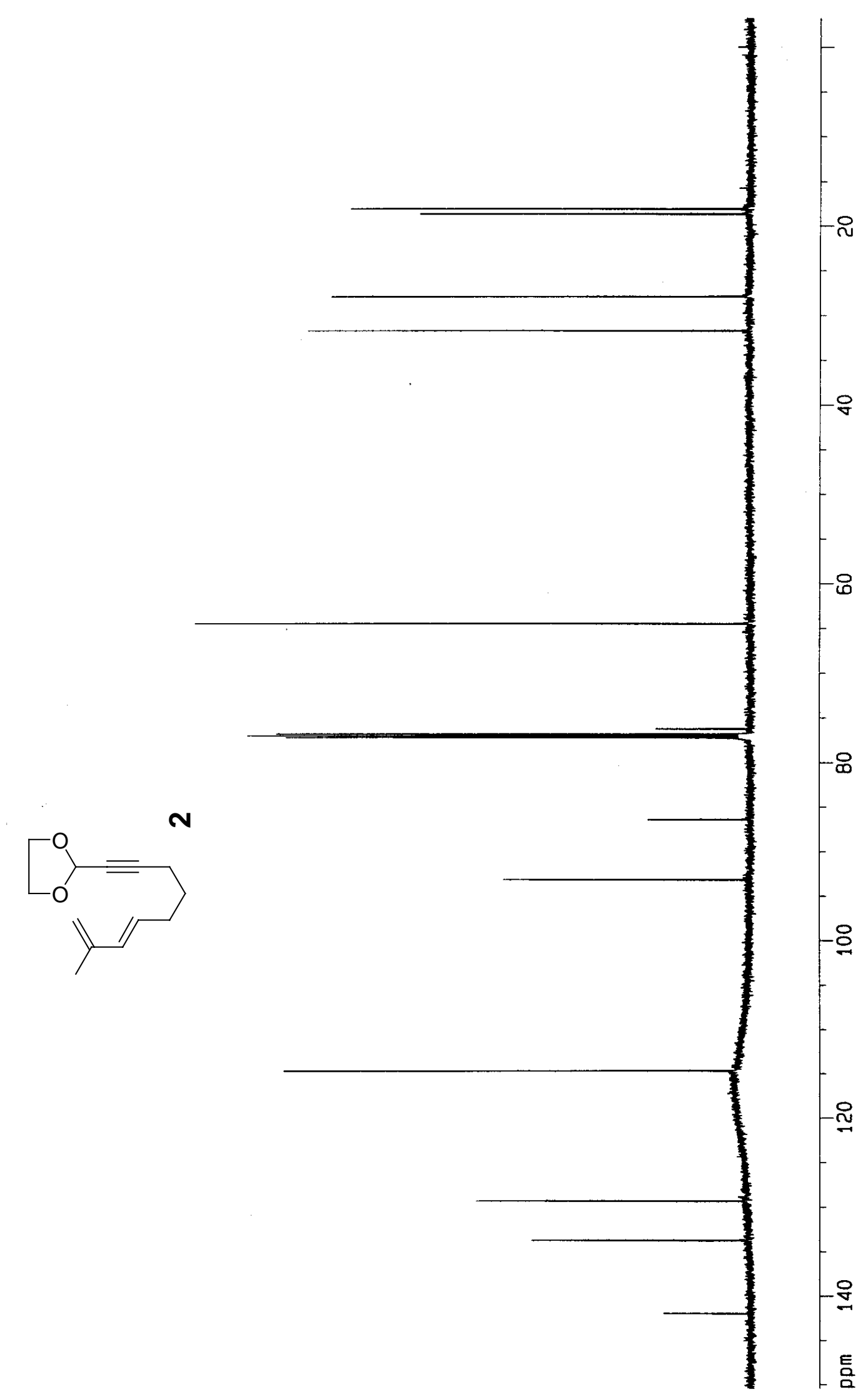


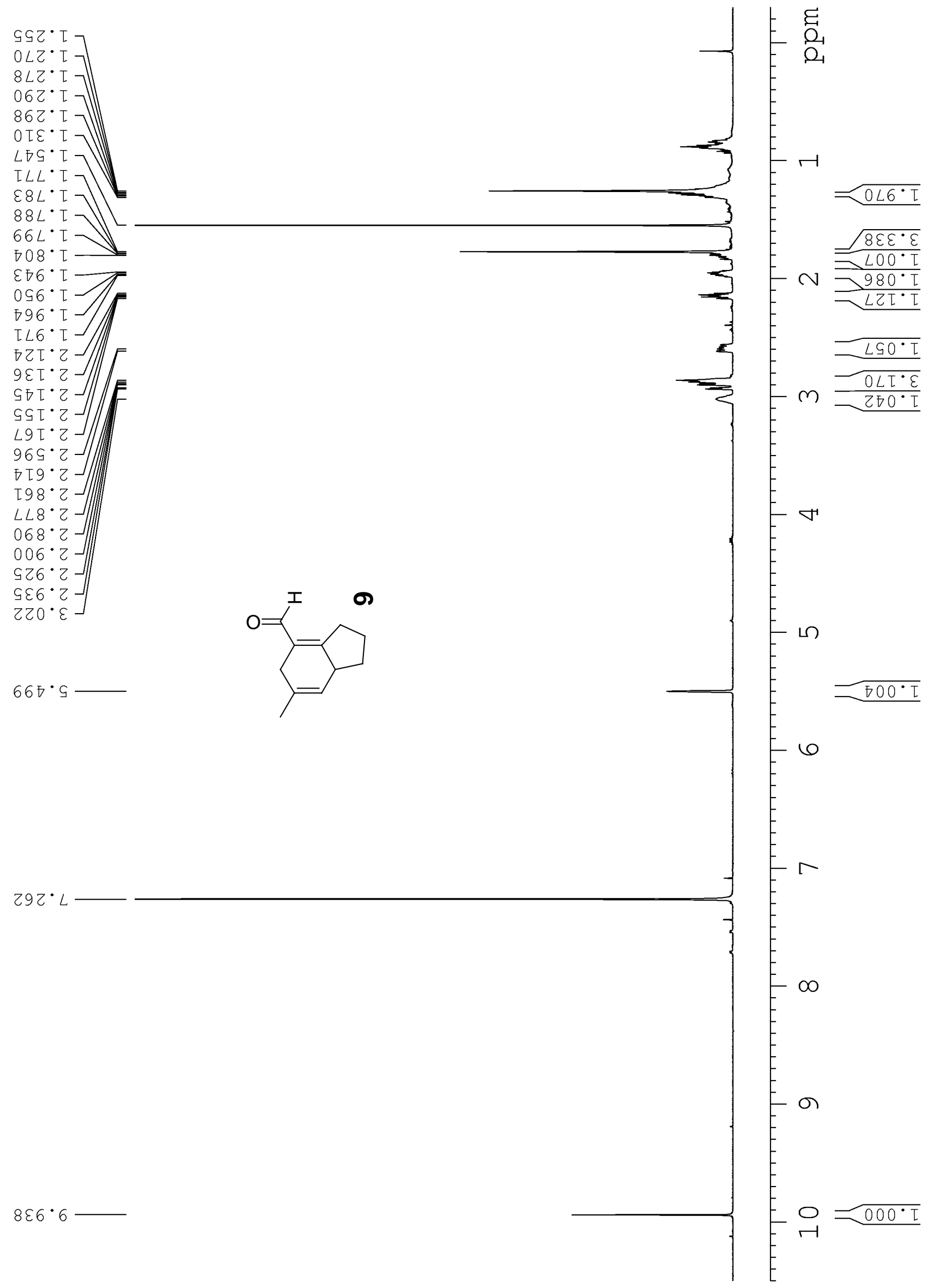




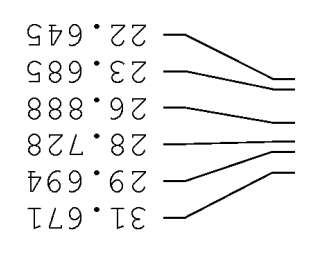

$9 \nabla 2 \cdot 9 \nabla \longrightarrow$

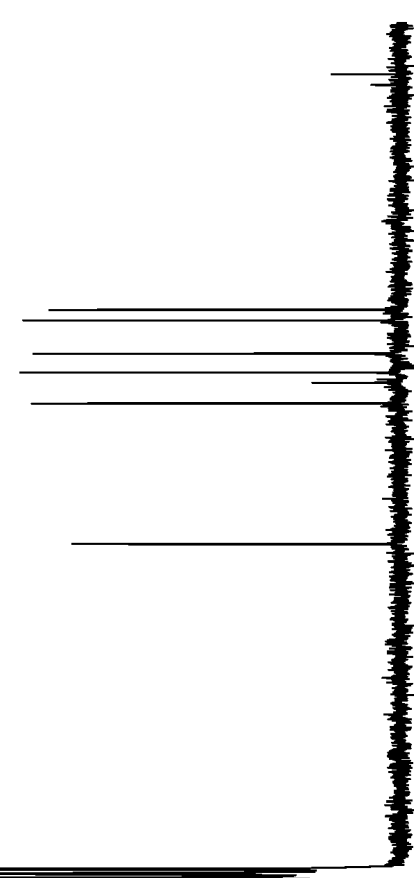

$\nabla \varepsilon S \cdot 0 z I$

$8 L Z \cdot 8 Z I-$

$289^{\circ}$
$700^{\circ}$
$80 Z \cdot L L D$
$\angle Z D^{\circ} \cdot L L$

$89 \tau \cdot \varepsilon \varepsilon \tau$

$200 \cdot 99 I$

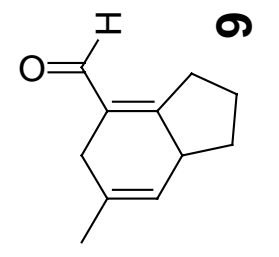

$60 L \cdot 06 I$

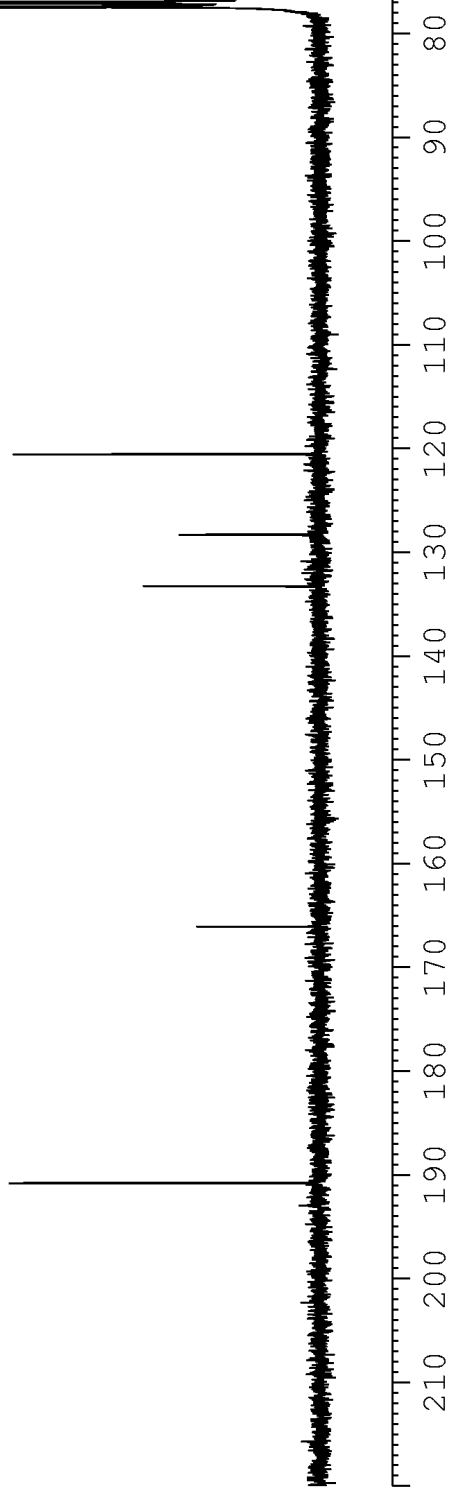




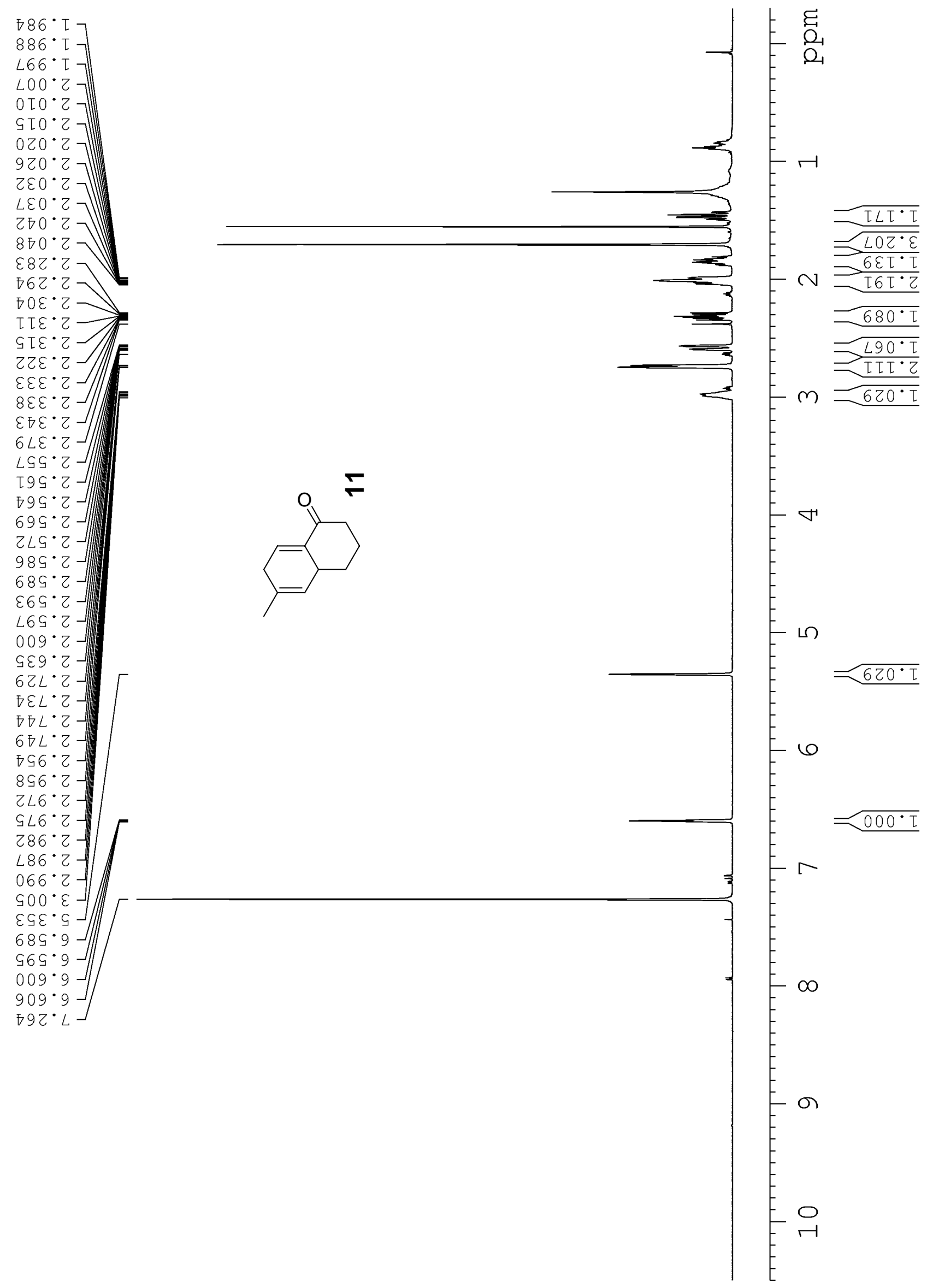




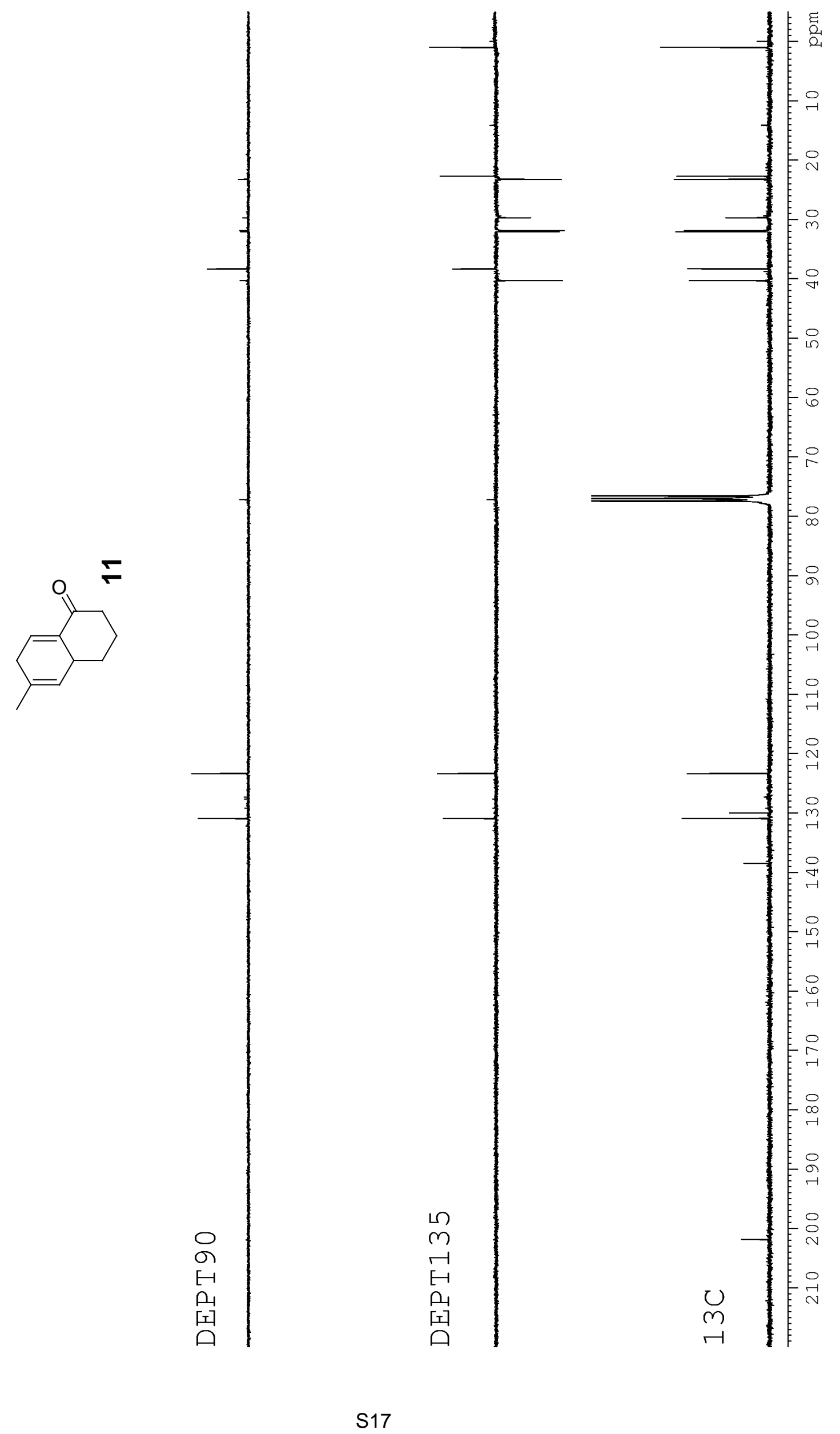

